\title{
Luminous Intensity Control System Based on ZigBee Wireless Sensor Networks
}

\author{
PEI Yan-ming ${ }^{\mathrm{a},{ }^{* 1}}$, LIU Yun-hong ${ }^{\mathrm{a}, * 2}$ \\ ${ }^{a}$ Department of Electrical Engineering, Dalian University of Technology, Dalian, China
}

\begin{abstract}
This paper introduces an energy conservation luminous intensity control system based on ZigBee sensor networks for smart home. The study is conducted to reduce unnecessary energy consumption by changing the luminous intensity of white LED according to the light coming into the room. A detecting circuit connected to the pin of CC2430 is set on the working face to collect the real-time luminous intensity signal. Then the ZigBee module adjusts the PWM signal to drive the white LEDs aiming at keeping a fixed and appropriate luminous intensity (e.g. 500Lux). Meanwhile, it transmits the data to the ZigBee coordinator through ZigBee wireless network. The monitoring PC get the data through the serial communication interface and display the states of the lights. Administrators can turn on or off the lights by clicking on the screen. This system can realize monitoring and controlling all the lights wirelessly so that users can save a lot energy and staff members. The system has been proved to be effective and convenient.
\end{abstract}

Index Terms: ZigBee; Smart Home; LabVIEW; CC2430

(C) 2012 Published by MECS Publisher. Selection and/or peer review under responsibility of the Research Association of Modern Education and Computer Science

\section{Introduction}

Since the energy crisis is becoming more and more serious, it's necessary to restrain the waste of electricity. This system can lower the luminous intensity of white LED automatically, when there's enough sunshine irradiating into the room. The administrator can remotely turn on or off anyone light in the control center by clicking the buttons on a computer, which is running a monitoring program in LabVIEW. It is convenient and needn't communication cost.

\section{ZigBee Technology Overview}

The ZigBee is a new wireless network technology which has the advantages in extremely low cost, reliable data transfer, short time-delay and a long battery life. There're mainly three kinds of topologies for ZigBee wireless network, which is shown in Fig.1.

\footnotetext{
* Corresponding author:

| E-mail address: ${ }^{* 1}$ peiyanming@ foxmail.com ${ }^{* 2}$ lyunhong2003@yahoo.com.cn
} 


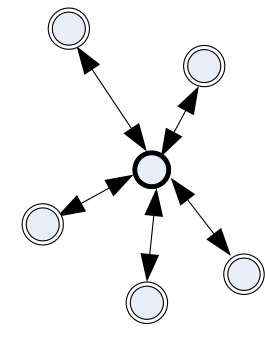

Coordinator

(a)Star topology
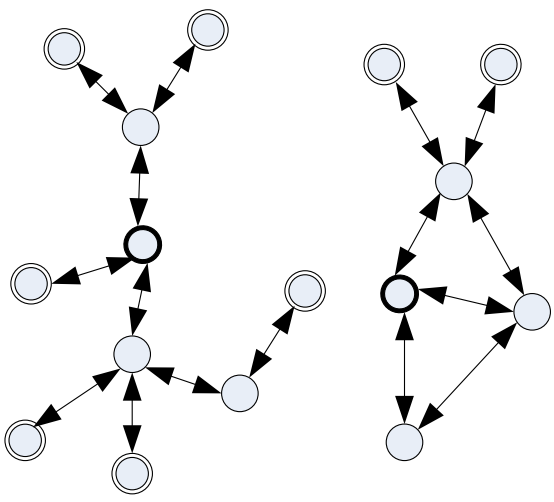

End Device

(b) Tree topology

(c)Net topology

Fig. 1. Topologies of ZigBee network

According to the ZigBee protocol, the ZigBee signal can at least transmit 30 meters in a house. Considering the advantages of simple construction, easy connection and low cost, we choose the star topology to build the network [1]. The structure of ZigBee devices is shown in Fig.2. There're two kinds of ZigBee devices: Full Function Devices (FFD) and Reduced Function Devices (RFD). The FFD device works as a ZigBee coordinator or router in a ZigBee network and the RFD device can only be the ZigBee end device [2].

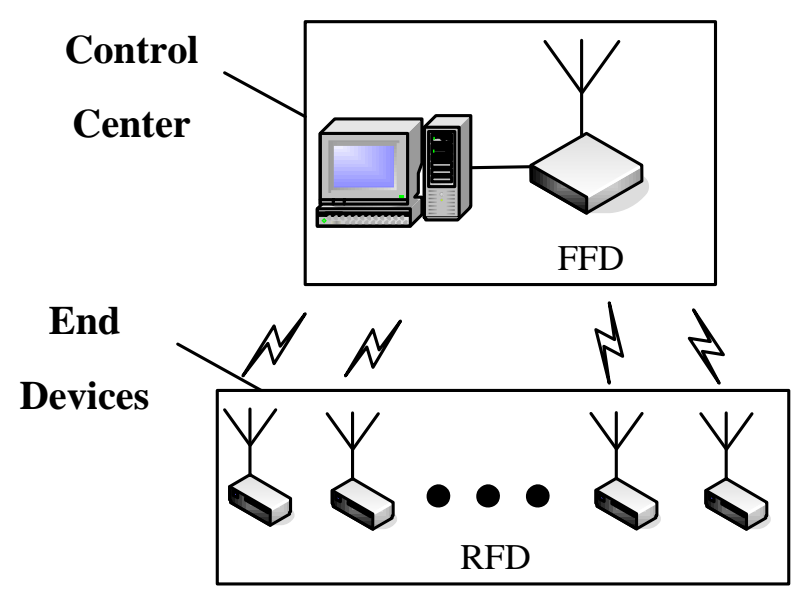

Fig. 2. Structure of ZigBee devices

The model of ZigBee standard stack architecture is shown in Fig.3 [3]. The ZigBee alliance defines the Network (NWK) Layer and the Application (APL) Layer. The Physical (PHY) Layer and Medium Access Control (MAC) Layer are defined by the IEEE 802.15.4. ZigBee adopts Direct Sequence Spread Spectrum (DSSS) technology and works in the $2.4 \mathrm{GHz}$ (available worldwide), $915 \mathrm{MHz}$ (North America and Australia) and $868 \mathrm{MHz}$ (Europe) ISM band. 


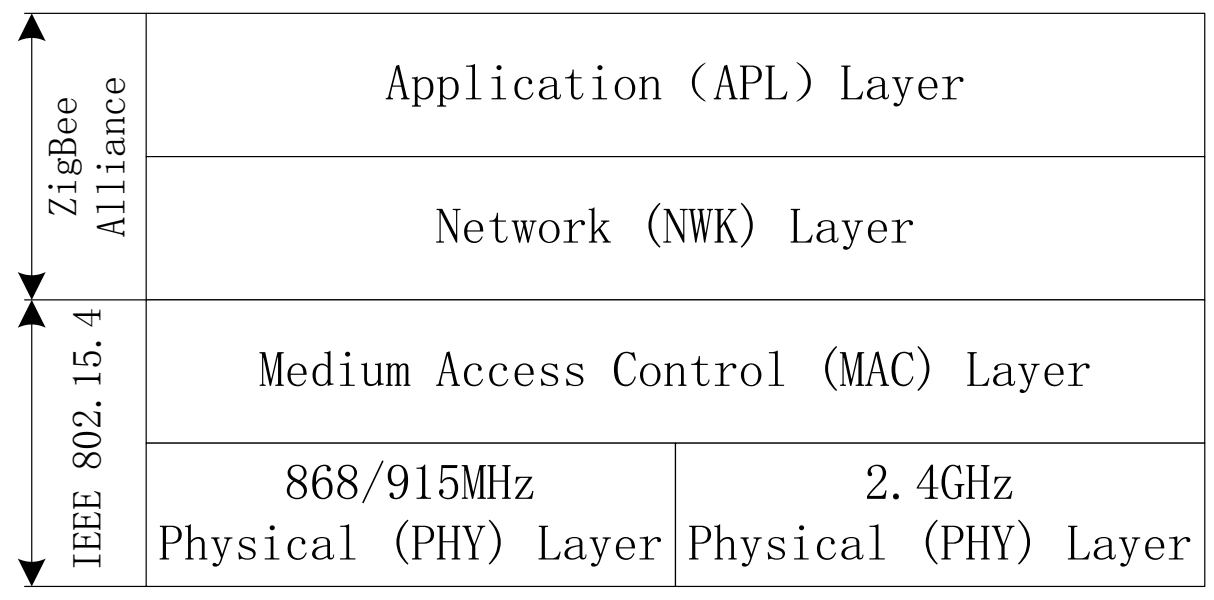

Fig. 3. Model of ZigBee standard stack architecture

\section{Hardware Design}

\subsection{RF Module}

The system uses TI's chip CC2430 to form a ZigBee wireless network. CC2430 is a System-on-Chip (SOC) solution for IEEE 802.15.4 and ZigBee applications [5]. It's a combination of a CC2420 RF transceiver and an industry-standard enhanced 8051 microcontroller. It only needs several resistors, capacitors and inductances to form a RF module.

\subsection{ZigBee Coordinator}

The ZigBee coordinator establishes the ZigBee wireless network, manages the other nodes and controls the communication between computer and the network [6]. In this system, we select UART0 as the serial communication interface to transmit data with computer. The structure block diagram of ZigBee coordinator is shown in Fig.4 [7].

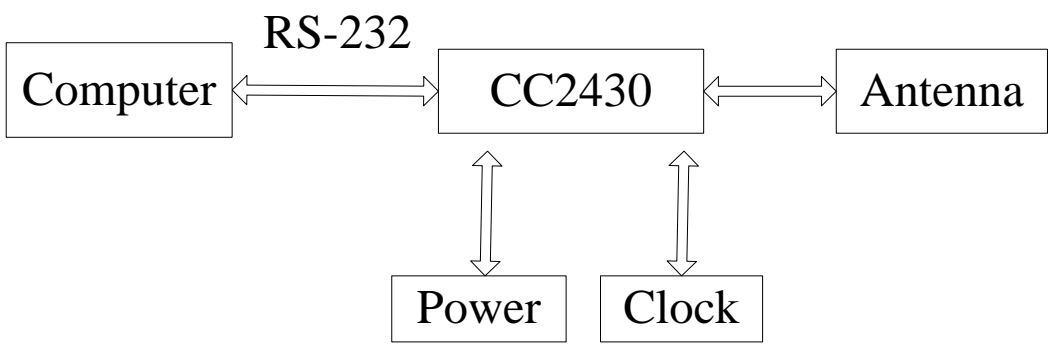

Fig. 4. Structure block diagram of ZigBee coordinator 


\subsection{ZigBee End Device}

ZigBee end device is also named sensor node. It consists of CC2430, reset circuit, antenna, power source and so on. Fig.5 shows the structure block diagram of ZigBee end devices.

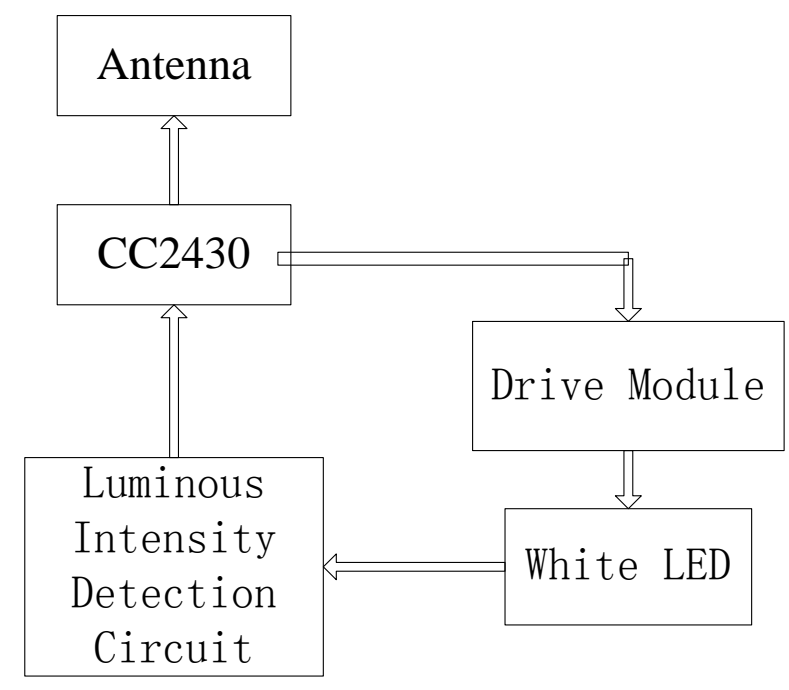

Fig. 5. Structure block diagram of ZigBee end devices

A photoresistance is used to detect luminous intensity on the desktop. Voltage on the photoresistance will change as soon as the luminous intensity changes, so the detection circuit transmits this information to the Analog to Digital Converter (ADC) of CC2430. CC2430 receives the digital signal and decide how to control the output Pulse Width Modulation (PWM), which can change the white LED luminous intensity. Below is the control strategy of luminous intensity:

- The actual luminous intensity is larger than the standard value, CC2430 decreases the duty cycle of PWM drive signal.

- The actual luminous intensity is smaller than the standard value, CC2430 increases the duty cycle of PWM drive signal.

In the system, white LED works in a constant current drive signal. LED will become brighter, if the duty cycle of PWM drive signal becomes larger. In this way, the luminous intensity on the desk will keep the standard value no matter how the environment changes.

\section{Software Design}

\subsection{Lower Computer}

In the software design of the system, we use the IAR Embedded Workbench as a compiler and C language to programme the application layer of ZigBee stack. The data processing flow is shown in Fig.6. 


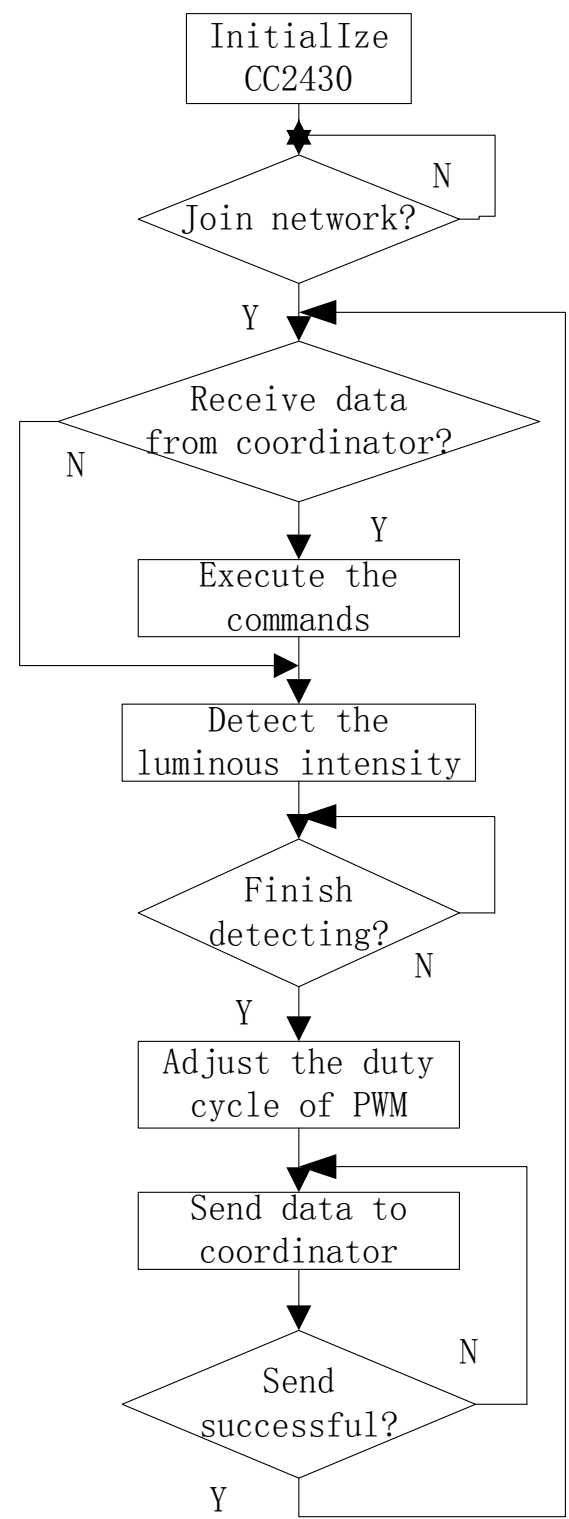

(a)Flow chart of the end devices

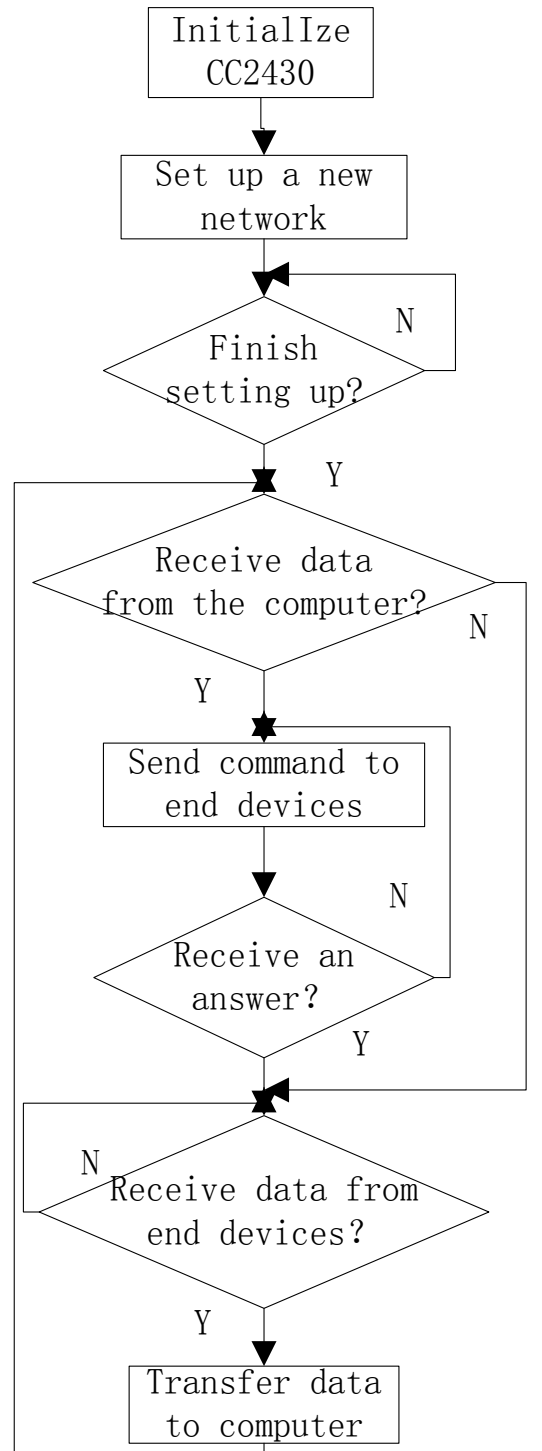

(b) Flow chart of the coordinator

Fig. 6. The data processing flow

To communicate with the control center, we make the coordinator to work in the module of full-duplex communication. The baud rate is calculated according to (1).

$$
\text { Bandrate }=\frac{\left(256+B A U D_{-} M\right) \times 2^{\text {BAUD_E }}}{2^{28}} \times \mathrm{F}
$$


Through the serial port, CC2430 can connect with computer via RS-232 connector. The messages we get when CC2430 is detecting the luminous intensity is shown in Fig.7.

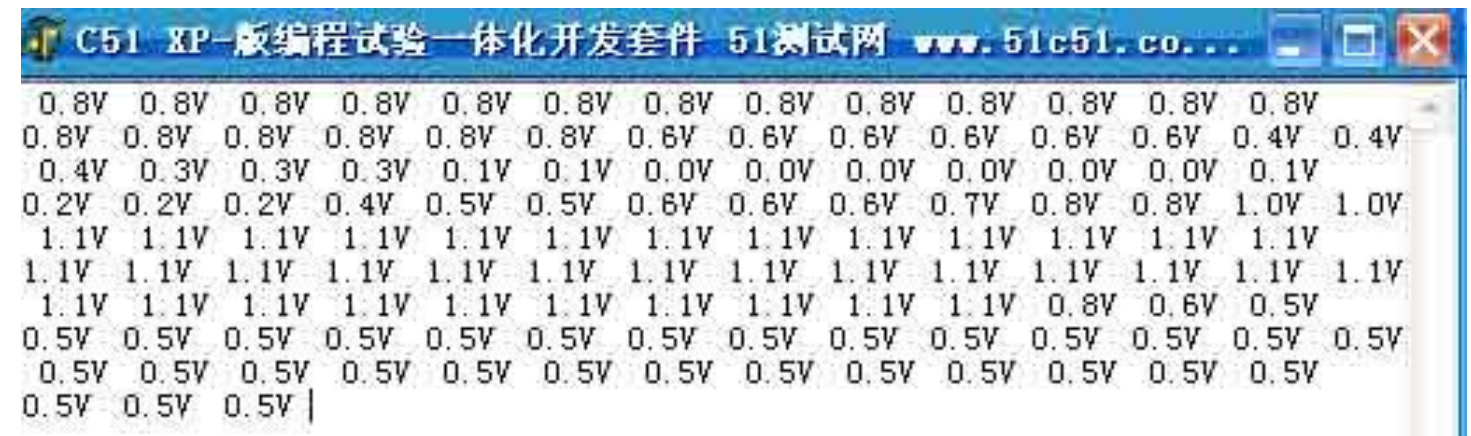

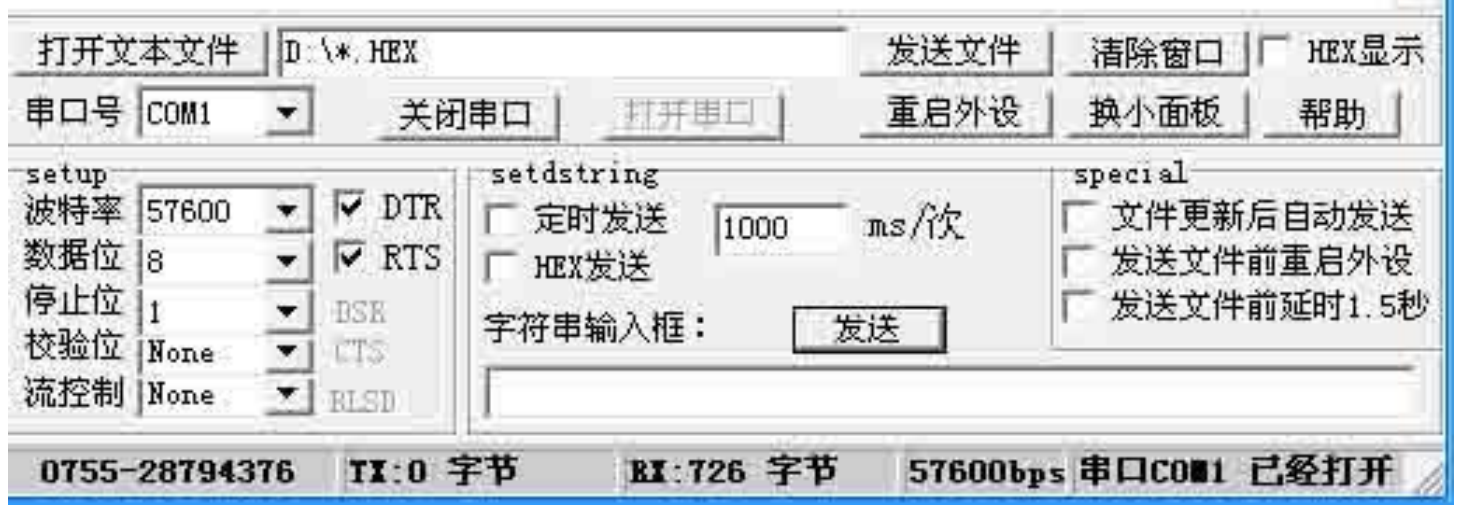

Fig. 7. Message between PC and CC2430

\subsection{Upper Computer}

To set up a monitoring system, LabVIEW, a high-level graphical development environment designed specifically for measurement and control applications, is adopted.

\section{1) Serial Read and Write:}

In order to communicate with the ZigBee coordinator, the serial read and write is necessary. On the block diagram, the Configure Serial Port sets the configuration parameters. The front panel displays the controls used to configure the basic parameters, such as baud rate, data bits, and parity. Fig.8 shows the block diagram and front panel in the monitoring system. All of the data which the ZigBee network wants to send and receive must use the serial read and write part. The parameters are set as follows. 


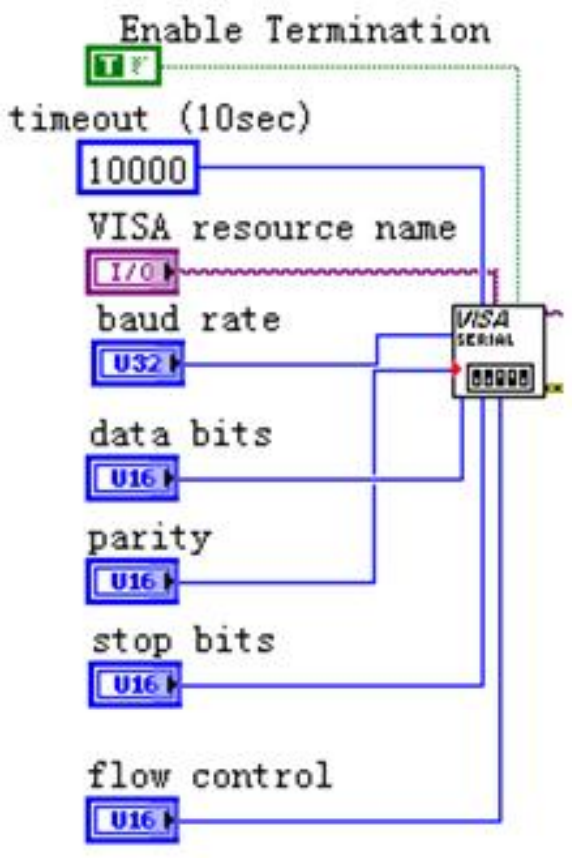

(a)Block diagram

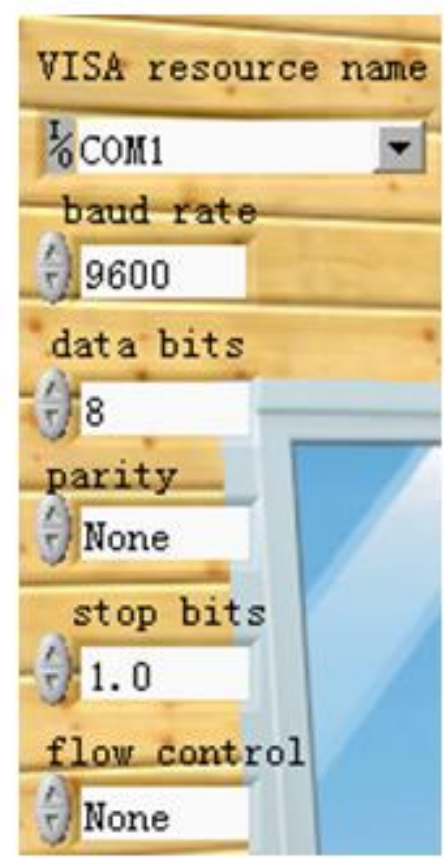

(b) Front panel

Fig. 8. Serial read and write

\section{2) Accounts Management:}

The monitoring system can turn on or off any white LED, so only the administrators can log in and control the lights. In this condition, we program an account management part in the software. The administrator has to input his user name, password and verification code before logging on. The block diagram is shown in Fig.9.

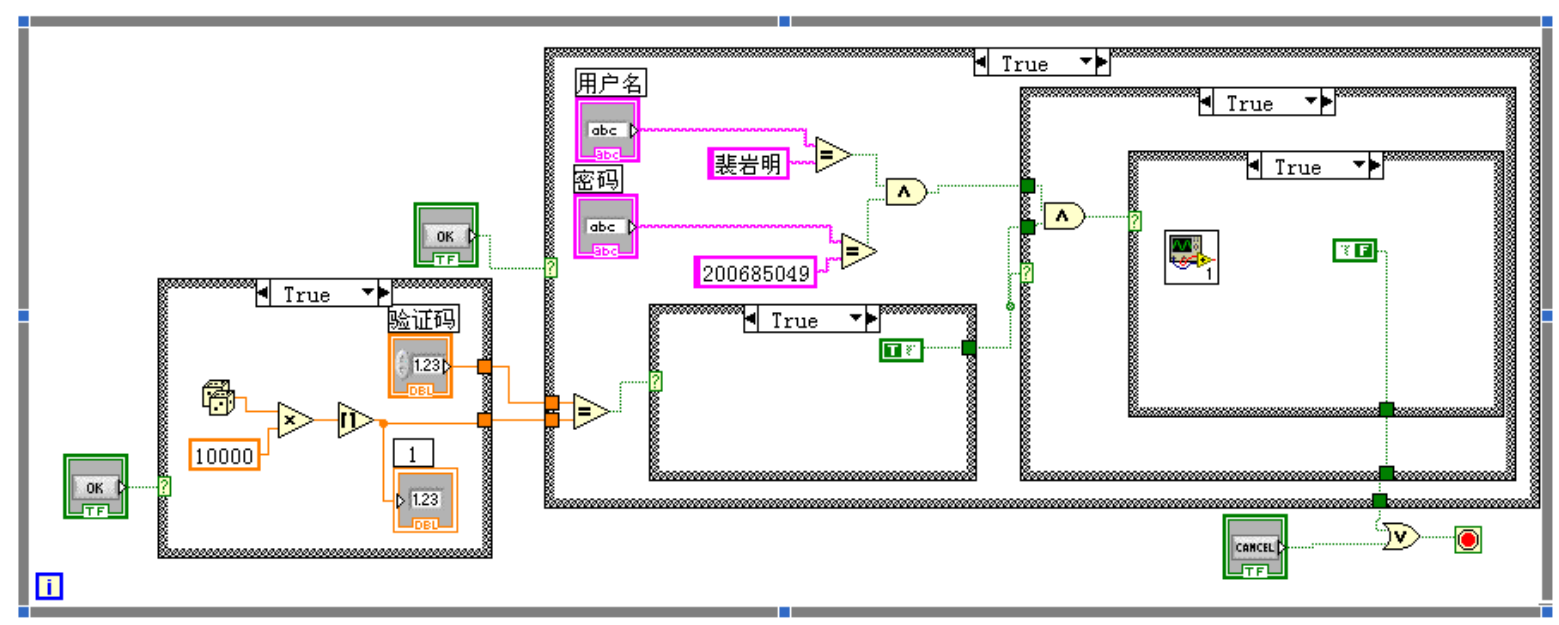

Fig. 9. The block diagram of accounts management part 


\section{3) State Machine:}

State machine is a module of program design in LabVIEW. It mainly includes several cases inside a while loop. So the Mealy state machine is quite suit for this monitoring system, because it's made up of three states listed below.

\section{a) Initialization:}

In this state, the LEDs are forced to die out, the button are forced to shut and the luminous intensity chart is forced to clear.

\section{b) Display:}

According to what the serial read from the ZigBee coordinator, it displays the state of LEDs and the luminous intensity in the front panel.

\section{c) Send command:}

This is a state for the computer to send command to the ZigBee end devices. If the administrator clicks on 'Button', the PC will send a command to ZigBee coordinator which is connected with it via the serial port.

The monitoring interface is shown as Fig.10.

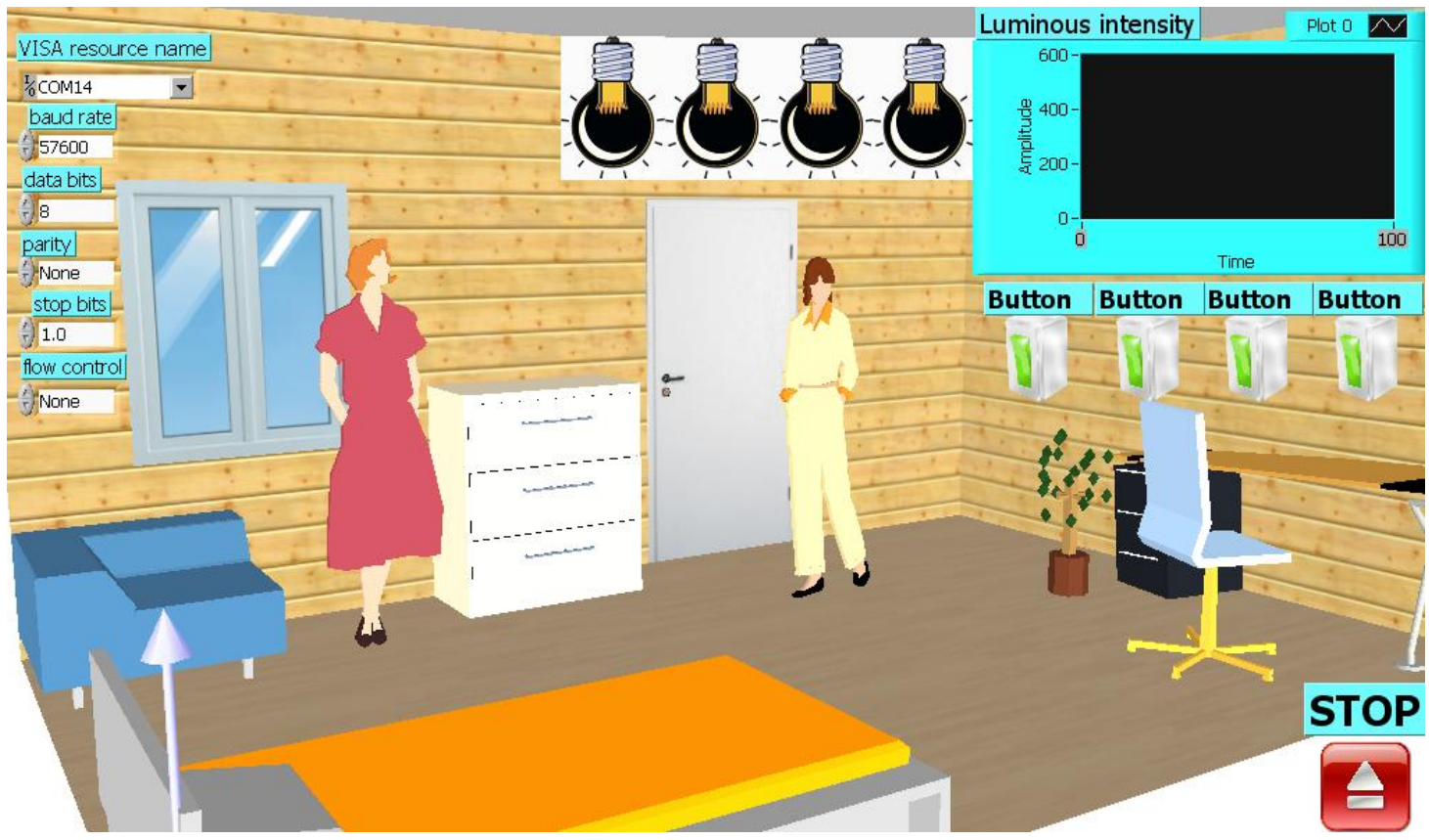

Fig. 10. Monitoring interface

\section{Conclusion}

In this paper, we proposed a system which has the advantages of energy saving, simple structure, high reliability and high practicality. It's typical application for IEEE 802.15.4. Making use of LabVIEW, we program a monitoring system to help administrators to manage all of the lights remotely in the control center. It's convenient and useful. 


\section{References}

[1] ZigBee Alliance, ZigBee-2007 Specification, Oct. 2007.

[2] Paolo Baronti, Prashant Pillai, Vince W.C.Chook, Stefano Chessa, Alberto Gotta and Y. Fun Hu, "Wireless sensor networks: A survey on the state of the art and the 802.15.4 and ZigBee standards," Computer Communications 30 (2007) 1655-1695.

[3] K. Akkaya, M. Younis, "A survey on routing protocols for wireless sensor networks", Ad Hoc Networks 3 (3), 2005 ,pp.325-349.

[4] M. Yin and L. Wang, "Design of general wireless communications module based on zigbee,"Computer and Communications(交通与计算机), vol.5, pp.110-112, 2006.

[5] Texas Instruments. Datasheet. CC2430.www.ti.com.

[6] Dechuan Chen, Meifang Wang, "A Home Security ZigBee Network for remote Monitoring Application," The IET International Conference on Wireless Mobile \& Multimedia Networks , pp. 304-307, 2006.

[7] Zhou Yiming, Yang Xianglong, Guo Xishan, Zhou Mingang, Wang Liren, "A Design of Greenhouse Monitoring \& Control System Based on ZigBee Wireless Sensor Network," The International Conference on Wireless Communications, Networking and Mobile Computing, pp.2563-2567, 2007.

[8] Tang Lichan, Qi Liang, Wang Qingdong, Chen Jianghong, “Temperature Supervisory System by Wireless Remote Communication Based on LabVIEW,” Journal of Shanghai Electric Technology(上海电气技术), vol. 2 No. 1, pp.20-24, 2009. 